\title{
Effects of chronic prazosin, an alpha-1 adrenergic antagonist, on anxiety-like behavior and cortisol levels in a chronic unpredictable stress model in zebrafish (Danio rerio)
}

\author{
Michael P O'Daniel ${ }^{1}$, Maureen L Petrunich-Rutherford ${ }^{\text {Corresp. } 1}$ \\ 1 Department of Psychology, Indiana University Northwest, Gary, Indiana, United States \\ Corresponding Author: Maureen L Petrunich-Rutherford \\ Email address: mlpetrun@iun.edu
}

Post-traumatic stress disorder (PTSD) is often associated with significant neuroendocrine dysfunction and a variety of other symptoms. Today, there are limited efficacious treatment options for PTSD, none of which directly target the dysfunction observed with the hypothalamic-pituitary-adrenal (HPA) axis. The development of new pharmacological treatments is expensive and time consuming; thus, there is utility in repurposing compounds already approved for use in other conditions. One medication in particular that has shown promise for the alleviation of PTSD symptoms is prazosin, an alpha-1 adrenergic receptor antagonist used to treat hypertension. While there have been many studies indicating the efficacy of prazosin in the treatment of PTSD symptoms, no studies fully elucidate mechanisms elicited by this treatment, nor is it clear if prazosin normalizes neuroendocrine dysfunction associated with trauma exposure. The use of zebrafish (Danio rerio) has been growing in popularity, in part, due to the homology of the stress response system with mammals. In this study, the zebrafish model was utilized to determine behavioral and biological changes induced by chronic unpredictable stress (CUS) and how these effects could be modulated by chronic prazosin treatment. The results indicated that $7 \mathrm{~d}$ of CUS increased anxiety-like behavior in the novel tank test and decreased basal levels of cortisol. Chronic (7d) prazosin treatment decreased anxiety-like behaviors overall but did not appear to affect CUS-induced changes in behavior and basal cortisol levels. This suggests that the clinical effectiveness of prazosin may not normalize dysregulated stress responses prevalent in many patients with PTSD, but that prazosin-induced relief from anxiety in stress-related conditions may involve an alternative mechanism other than by normalizing neuroendocrine dysfunction. 
1 Effects of chronic prazosin, an alpha-1 adrenergic antagonist, on anxiety-like

2 behavior and cortisol levels in a chronic unpredictable stress model in

3 zebrafish (Danio rerio)

4

$5 \quad$ Michael P. O’Daniel ${ }^{1}$ and Maureen L. Petrunich-Rutherford ${ }^{1}$

6

$7{ }^{1}$ Department of Psychology, Indiana University Northwest, Gary, Indiana, USA

8

9 Corresponding Author:

10 Maureen L. Petrunich-Rutherford

11 Email address: mlpetrun@iun.edu

12

13

14

15

16

17

18

19

20

21

22 


\section{Abstract}

Post-traumatic stress disorder (PTSD) is often associated with significant neuroendocrine

dysfunction and a variety of other symptoms. Today, there are limited efficacious treatment

options for PTSD, none of which directly target the dysfunction observed with the hypothalamicpituitary-adrenal (HPA) axis. The development of new pharmacological treatments is expensive and time consuming; thus, there is utility in repurposing compounds already approved for use in other conditions. One medication in particular that has shown promise for the alleviation of PTSD symptoms is prazosin, an alpha-1 adrenergic receptor antagonist used to treat

31 hypertension. While there have been many studies indicating the efficacy of prazosin in the treatment of PTSD symptoms, no studies fully elucidate mechanisms elicited by this treatment, nor is it clear if prazosin normalizes neuroendocrine dysfunction associated with trauma exposure. The use of zebrafish (Danio rerio) has been growing in popularity, in part, due to the homology of the stress response system with mammals. In this study, the zebrafish model was utilized to determine behavioral and biological changes induced by chronic unpredictable stress

37 (CUS) and how these effects could be modulated by chronic prazosin treatment. The results indicated that $7 \mathrm{~d}$ of CUS increased anxiety-like behavior in the novel tank test and decreased basal levels of cortisol. Chronic (7d) prazosin treatment decreased anxiety-like behaviors overall but did not appear to affect CUS-induced changes in behavior and basal cortisol levels. This suggests that the clinical effectiveness of prazosin may not normalize dysregulated stress responses prevalent in many patients with PTSD, but that prazosin-induced relief from anxiety in stress-related conditions may involve an alternative mechanism other than by normalizing neuroendocrine dysfunction. 


\section{Introduction}

Post-traumatic stress disorder (PTSD) is a disorder that inhibits day-to-day functionality

due to a plethora of disrupting symptoms including flashbacks, vivid nightmares, mood alterations, and hypervigilance (Bisson, Cosgrove, Lewis, \& Robert, 2015). According to the World Health Organization (WHO) World Mental Health Surveys, the overall lifetime prevalence of PTSD is estimated to be around 3.9\%, increasing to about $5.6 \%$ of trauma-exposed individuals (Koenen et al., 2017). The most common traumas associated with PTSD are interpersonal traumas, including rape and other types of sexual assault (Kessler et al., 2017). Current evidence-based treatment plans for PTSD include cognitive behavioral therapy and pharmacological options, mainly serotonin reuptake inhibitors (Lancaster, Teeters, Gros, \& Back, 2016); however, many cases are resilient to or respond ineffectively to first-line treatments (Foa, Keane, Friedman, \& Cohen, 2009). It appears that there is a growing trend of prescribing benzodiazepines for the management of PTSD symptoms, at least in U.S. active duty service members (Loeffler, Coller, Tracy, \& Derderian, 2018); however, this practice is associated with concerning outcomes, such as increased suicide risk (Deka et al., 2018). In addition, it is well known that chronic use of benzodiazepines is associated with physiological dependence and subsequent withdrawal symptoms upon treatment discontinuation (Pétursson, 1994).

Due to the resilience to treatment and concerning growth in use of sedative prescription medications, the need for novel treatments for PTSD has become apparent. Because of the exorbitant cost of effective novel drug synthesis and testing, there has been growing interest in the repurposing of compounds already approved by the Food and Drug Administration in the treatment of other conditions (Papapetropoulos \& Szabo, 2018). Prazosin, an alpha-1 adrenergic receptor antagonist originally utilized in the treatment of hypertension, has been shown to 
69 alleviate clinical symptoms of PTSD (Ahmadpanah et al., 2014; De Berardis et al., 2015; Green, 70 2014; Koola, Varghese, \& Fawcett, 2014; Simon \& Rousseau, 2017; B. Singh, Hughes, Mehta,

71 Erwin, \& Parsaik, 2016; Writer, Meyer, \& Schillerstrom, 2014). However, there have been few

72 investigations into the mechanisms behind the clinical efficacy of this compound, particularly

73 involving physiological symptoms associated with chronic stress exposure.

The physiological stress response in mammals is largely controlled by the hypothalamic-

75 pituitary-adrenal (HPA) axis. The HPA axis acts in a negative feedback loop, wherein the hypothalamus signals the pituitary gland via corticotropin-releasing hormone $(\mathrm{CRH})$ to stimulate the release of adrenocorticotropic hormone (ACTH) into the bloodstream. ACTH then acts peripherally to stimulate the release of glucocorticoids (e.g., cortisol, corticosterone) from the adrenal glands to mobilize the body's resources to deal with a stressor. Then, cortisol binds to glucocorticoid receptors in the hypothalamus, pituitary gland, and other upstream brain structures to attenuate the stress response. Studies indicate that the HPA axis is dysregulated in patients with PTSD (Mason, Giller, Kosten, Ostroff, \& Podd, 1986; Pervanidou \& Chrousos, 2010; Wichmann, Kirschbaum, Böhme, \& Petrowski, 2017; Yehuda et al., 1990). There is evidence that prazosin helps to normalize HPA dysfunction in subjects in the early stages of alcohol withdrawal (Fox et al., 2012); however, it is unknown if prazosin would similarly alleviate HPA dysfunction associated with chronic stress exposure. Furthermore, the exact direction of chronic stress-induced dysregulation (i.e., upregulation or downregulation) is complex and likely dependent on a number of factors, such as biological sex and early life stress exposure (Dunlop \& Wong, 2019). Thus, a more complete understanding of individual factors affecting stress-induced alterations in HPA functioning can be examined with animal models. 
92 rerio) has been asserted as a viable model for stress related research because of the similarities in

93 the physiological stress response (Clark, Boczek, \& Ekker, 2011). The hypothalamic-pituitary-

94 interrenal (HPI) axis is considered to be the zebrafish analogue to the mammalian HPA axis

95 (Nesan \& Vijayan, 2013; Wendelaar Bonga, 1997). In addition, zebrafish have been growing in

96 popularity for translational research due to genetic and physiological similarities to mammals in

97 stress and anxiety-like behavioral responses (Caramillo, Khan, Collier, \& Echevarria, 2015).

98 Zebrafish exposed to chronic unpredictable stress (CUS) may facilitate a better understanding of

99 factors that may convey vulnerability to behavioral disorders that affect humans, such as major

100 depressive disorder (Fulcher, Tran, Shams, Chatterjee, \& Gerlai, 2017) and PTSD (Caramillo et

101 al., 2015; Stewart, Yang, Nguyen, \& Kalueff, 2014). The stressors that are utilized in the CUS

102 zebrafish model vary in intensity, duration of stress, and type of stress and include mechanical,

103 chemical, and temperature changes. The stressors are randomized and are administered at

104 different times. Several studies have indicated that CUS modeling in zebrafish elicits anxiety-

105 like behaviors in a variety of testing paradigms (Chakravarty et al., 2013; Fulcher et al., 2017;

106 Marcon et al., 2016, 2018; Piato et al., 2011; Song et al., 2018). CUS also increases whole-body

107 cortisol levels (Manuel et al., 2014; Marcon et al., 2016, 2018; Piato et al., 2011; Song et al.,

108 2018), although in one report, CUS was shown to increase basal cortisol levels only in male fish

109 but did not significantly alter basal cortisol levels in female fish (Rambo et al., 2017). Thus, the

110 CUS model in zebrafish could be used to examine the efficacy of repurposed compounds and

111 clarify the mechanisms by which these compounds could alleviate physiological dysfunction

112 associated with stress-related conditions. 
114 reported in the literature (Manuel et al., 2014; Marcon et al., 2016, 2018; Piato et al., 2011;

115 Rambo et al., 2017) to replicate the effects of the protocol on anxiety-like behavior and basal

116 cortisol levels. Then, in another experiment, chronic treatment with either prazosin or vehicle

117 followed a week of CUS to examine whether prazosin would reverse any CUS-induced changes

118 in hormones or behavior. It was hypothesized that CUS would increase basal levels of cortisol

119 and elicit increases in anxiety-like behavior, as evidenced by increased freezing and decreased

120 exploratory behavior in the novel tank test. It was also expected that chronic prazosin treatment

121 would normalize both neuroendocrine and behavioral alterations observed after CUS exposure.

122 The results from the current study could provide evidence for the mechanism of prazosin's

123 clinical efficacy and suggest that it may be a viable treatment option for individuals with HPA

124 dysfunction associated with stress-related conditions, such as PTSD.

125

\section{Methods and Materials}

Animals and housing

128 Wild-type, adult mixed-sex zebrafish (total $\mathrm{N}=122$ ) were purchased from Carolina

129 Biological Supply (Burlington, NC). Upon delivery, zebrafish were randomly placed into

130 housing tanks and allowed to acclimate to the facility for at least one week before any

131 experimental procedures were initiated (Dhanasiri, Fernandes, \& Kiron, 2013). Zebrafish were

132 housed in a stocking density of 5-7 fish per liter in 1.8L tanks and maintained in a two-shelf,

133 stand-alone zebrafish housing rack purchased from Aquaneering (San Diego, CA). Fish were

134 maintained on a 14h:10h light:dark cycle, with water kept at $27 \pm 1^{\circ} \mathrm{C}$ and $\mathrm{pH}$ of approximately

135 7.2. Other water quality parameters were measured biweekly, such as ammonia, nitrates, nitrites, 
136 alkalinity, and hardness, and were kept constant throughout the experiments. Fish were fed once

137 per day with flake food and once per day with dried shrimp ground to a powder with a mortar

138 and pestle. Feeding commenced around 9 a.m. each day, before any stress or drug procedures

139 were conducted, except for on days of data collection. The fish were not fed prior to the

140 behavioral testing. The total food weight given per day per fish approximated $4 \%$ of the average

141 fish body weight. All procedures were carried out by following established recommendations

142 (Harper \& Lawrence, 2011; National Research Council, 2011; Westerfield, 2000).

\section{Drugs and materials}

144 Prazosin hydrochloride was manufactured by TCI America and purchased from VWR

145 International (Radnor, PA). N-N-dimethylacetamide was manufactured by Frontier Scientific

146 and purchased from Fisher Scientific (Hampton, NH).

147 Experiment 1: Chronic unpredictable stress on anxiety-like behavior and basal cortisol levels

148 Upon arrival to the facility, fifty zebrafish were randomly allocated into four separate

149 1.8L housing tanks. After at least 7 days of acclimation to the facility, two tanks of fish were

150 randomly selected and subsequently exposed to the chronic unpredictable stress model for seven

151 days and the other two tanks of fish served as untreated, unstressed controls. On the day after the

152 completion of the chronic stress paradigm, fish from both control and stressed groups were

153 placed in the novel tank test one at a time to assess anxiety-like behavior. Fish were immediately

154 euthanized after the behavioral assessment and decapitated to assess basal levels of trunk

155 cortisol. At the end of the experiment, $\mathrm{N}=25$ were exposed to chronic unpredictable stress and

$156 \mathrm{~N}=25$ were untreated for 7 days. 
159 behavior and basal cortisol levels

160 Upon arrival to the facility, seventy-two zebrafish were randomly allocated into eight

161 separate 1.8L housing tanks. After at least 7 days of acclimation to the facility, four tanks of fish

162 were randomly selected and subsequently exposed to the chronic unpredictable stress model for

163 seven days and the other four tanks of fish were not stressed (left unhandled) for seven days.

164 Then, two tanks of the stressed fish and two tanks of the non-stressed fish were treated with

165 prazosin for 30 minutes per day for the seven days following the CUS treatment. The other four

166 tanks were exposed to vehicle treatment for 30 minutes per day for seven days. On the day after

167 the completion of the drug or vehicle treatment, fish from all groups were placed in the novel

168 tank test one at a time to assess anxiety-like behavior. Fish were immediately euthanized after

169 the behavioral assessment and decapitated to assess basal levels of trunk cortisol. A total of three

170 fish died during the course of the procedures $(\mathrm{N}=2$ from the unstressed/vehicle-treated group

171 and $\mathrm{N}=1$ from the stressed/vehicle-treated group). At the end of the experiment, $\mathrm{N}=18$ were

172 exposed to $7 \mathrm{~d}$ chronic unpredictable stress followed by chronic (7d) prazosin, $\mathrm{N}=17$ were

173 exposed to $7 \mathrm{~d}$ chronic unpredictable stress followed by $7 \mathrm{~d}$ vehicle, $\mathrm{N}=18$ were untreated for 7

174 days and then exposed to chronic (7d) prazosin, and $\mathrm{N}=16$ were untreated for 7 days and then

175 exposed to vehicle for 7 days.

176 Chronic unpredictable stress (CUS) model

177 The chronic unpredictable stress (CUS) model was adapted from previously published

178 procedures (Marcon et al., 2016, 2018; Piato et al., 2011; Rambo et al., 2017). Seven different

179 types of stressors were ordered at random. Fish in the stressed group were exposed to two

180 stressors per day for seven days at random times between 9 a.m. and 4 p.m. (see Table 1 for 
181 schedule). The stressors included (1) tank changes (three times) in rapid succession, (2) cooling

182 home tank water abruptly to $23^{\circ} \mathrm{C}$ and maintaining that temperature with chilled system water for

18330 minutes before placing tank back on the system, (3) heating home tank water abruptly to $33^{\circ} \mathrm{C}$

184 and maintaining that temperature with heated system water for 30 minutes before placing tank

185 back on the system, (4) lowered water ( $1 \mathrm{~cm}$ depth for 15 minutes), (5) net chasing in home tank

186 (8 minutes chase, 15 minute rest, 8 minute chase), (6) crowding all fish from one home tank in

$187200 \mathrm{ml}$ system water (9-13 fish total, density of 45-65 fish per liter) in a $250 \mathrm{ml}$ beaker for 60

188 minutes, and (7) social isolation (individual fish were placed in $200 \mathrm{ml}$ system water in $250 \mathrm{ml}$

189 beakers separated by opaque dividers). Control fish were not stressed (not handled) and were not

190 removed from the system for 7 days.

191 Drug treatment

192 Fish undergoing chronic administration of prazosin were gently netted from the home

193 tank and individually placed into a $100 \mathrm{~mL}$ beaker containing $2 \mathrm{mg}$ prazosin dissolved in 100

194 microliters of N,N-dimethylacetamide and $50 \mathrm{~mL}$ of system water for 30 minutes (A. Singh,

195 Subhashini, Sharma, \& Mallick, 2013). This concentration was chosen due to the observed

196 anxiolytic behavior in the light-dark test displayed by the fish acutely exposed to prazosin (A.

197 Singh et al., 2013). In the current experiment, the drug exposure was repeated once daily for

198 seven days; fresh drug solution was prepared for each fish for each exposure from a concentrated

199 prazosin solution prepared at the beginning of the experiment. Subjects in the control group were

200 subjected to similar handling and conditions although only exposed to the vehicle (100

201 microliters of $\mathrm{N}, \mathrm{N}$-dimethylacetamide in $50 \mathrm{~mL}$ system water in a $100 \mathrm{~mL}$ beaker for 30 minutes

202 per day). Fresh vehicle solution was prepared each day. All fish were returned to their respective

203 home tanks after the treatment and placed back on the system between daily treatment sessions. 
204

205

206

207

208

209

210

211

212

213

214

215

216

217

218

219

220

221

222

223

224

225

226

Novel tank test (NTT)

On the day of the experiment (the day after the last episode of chronic stress for fish in Experiment 1 and the day after the last drug treatment for fish in Experiment 2), home tanks were removed from the system and moved into the experiment room adjacent to the housing facility. To minimize the impact of the stress of moving the tanks, fish were left to acclimate for at least 30 minutes before assessing behavior. The experimental room had the same lighting and temperature conditions as the housing room. Fish were individually netted and placed into a trapezoidal novel tank, the same size and dimensions as the home tanks $(15.2 \mathrm{~cm}$ height $\times 27.9$ $\mathrm{cm}$ top $\times 22.5 \mathrm{~cm}$ bottom $\times 7.1 \mathrm{~cm}$ width), for six minutes. The novel tank was filled with water from the system and was changed on each new day of data collection. The behavior of each fish was recorded and subsequently analyzed with BehaviorCloud motion-tracking software (Alia \& Petrunich-Rutherford, 2019; Aponte \& Petrunich-Rutherford, 2019; Pilehvar, Town, \& Blust, 2020). Total distance traveled $(\mathrm{cm})$ and mean ambulatory speed $(\mathrm{cm} / \mathrm{sec})$ were measured as markers of general motor activity; immobility duration $(\mathrm{sec})$, the number of entries to the top of tank, time spent in top $(\mathrm{sec})$, and distance traveled in the top $(\mathrm{cm})$ and were used as markers of anxiety-like behavior (Cachat et al., 2010; Egan et al., 2009; Wong et al., 2010). The top of the tank was defined as the top $50 \%$ (approximately $7 \mathrm{~cm}$ ) of the water column (total approximately $14 \mathrm{~cm}$ ). Behavioral data collection and euthanasia of the subjects occurred between 9:30 a.m. and 2:30 p.m.

\section{Euthanasia}

Immediately after the novel tank test, fish were netted from the novel tank and placed individually in a $50 \mathrm{~mL}$ beaker with approximately $30 \mathrm{~mL} 0.1 \%(100 \mathrm{mg} / \mathrm{L})$ clove oil in system water. Death occurred within 5 to 10 seconds of introduction to the solution and was determined 
227 upon visual examination for cessation of opercular (gill) movement and nonresponse to tactile

228 stimulation (Davis et al., 2015). The fish were then decapitated. The trunk samples were frozen

229 in individual $1.5 \mathrm{ml}$ tubes and stored at $-20^{\circ} \mathrm{C}$ for cortisol analysis.

230 Cortisol extraction and assay

231 The cortisol extraction and assay was done by slightly modifying previously published

232 procedures (Cachat et al., 2010; Canavello et al., 2011). In brief, trunk samples were thawed and

233 weighed, and subsequently homogenized with phosphate-buffered saline (PBS). Diethyl ether

234 was added to the homogenates and centrifuged for 15 minutes at $4^{\circ} \mathrm{C}$. The ether layer containing 235 cortisol was isolated in a separate tube. The addition of ether, centrifugation, and ether isolation

236 was repeated for a total of three times, collecting all three ether layers in one tube for each

237 sample. The ether was then dried under a light stream of air until only a yellow oil containing the

238 cortisol remained in each tube. The oil in each tube was reconstituted with PBS and refrigerated

239 overnight $\left(4^{\circ} \mathrm{C}\right)$. Cortisol was quantified via an enzyme-linked immunosorbent assay (ELISA) as

240 per the manufacturer's instructions (Salimetrics, State College, PA).

241 Data analysis

242 A priori sample size calculations were conducted using G*Power software (Faul,

243 Erdfelder, Lang, \& Buchner, 2007) using the following parameters: $d=0.95, \alpha=0.05$, power $=$

244 0.95. Effect size was based on the effects of chronic unpredictable stress and prazosin on anxiety

245 measures in previously published studies (Marcon et al., 2016; A. Singh et al., 2013).

246 Upon selection from the home tank on the day of the experiment, each fish was given a

247 sample number. Behavioral and cortisol analyses were conducted; sample numbers were then

248 matched with the treatment(s) and analyzed by group. Data are presented as the means and

249 standard errors of the mean (SEM) for each group. Raw data (see supplemental file 1) was 
250 processed using JASP software (University of Amsterdam, Amsterdam, The Netherlands,

251 https://jasp-stats.org/). For Experiment 1, overall (6-minute) behavioral variables, cortisol, and

252 trunk weights were compared by independent sample t-tests (with stress condition as the

253 independent variable) and one-minute bin data for behavioral variables were compared by

254 repeated-measures ANOVA. For Experiment 2, overall (6-minute) behavioral variables, cortisol,

255 and trunk weights were compared by two-way ANOVA analyses (with stress condition and drug

256 treatment as the independent variables) and one-minute bin data for behavioral variables were

257 compared by repeated-measures ANOVA. Tukey post-hoc analyses were conducted when

258 appropriate and Greenhouse-Geisser sphericity correction was made if Mauchly's test of

259 sphericity indicated a violation of the sphericity assumption for the repeated-measures ANOVA

260 tests. A significance level of $p<0.05$ was used as the criterion for results to reach statistical

261 significance.

262

263

Results

264 Experiment 1: Effects of seven days of chronic unpredictable stress (CUS) treatment on

265 behavioral measures in the novel tank test (NTT), basal levels of cortisol, and body weights in

266 adult zebrafish.

267 Motor activity in the novel tank test

268 A t-test for independent means indicated no significant effect of chronic unpredictable

269 stress on either the total distance traveled $(\mathrm{t}(48)=1.274, \mathrm{p}=0.209)$ or mean ambulatory speed

$270(\mathrm{t}(48)=1.077, \mathrm{p}=0.287)$ for the entire 6 minutes of the novel tank test (Table 2). When the total

271 distance data was broken down into six 60-second bins (Figure 1A) and analyzed with a

272 repeated-measures ANOVA, there was no effect of stress $(F(1,48)=1.551, p=0.219)$, a 
273 significant effect of time $(\mathrm{F}(3.877,186.085)=7.403, \mathrm{p}<0.001)$, but no interaction between stress

274 and time $(F(3.877,186.085)=0.452, p=0.765)$. For the mean ambulatory speed (Figure 1B),

275 again, there was no effect of stress $(F(1,48)=0.934, p=0.339)$, a significant effect of time

$276(\mathrm{~F}(4.077,195.709)=16.657, \mathrm{p}<0.001)$, but no interaction between stress and time

$277(\mathrm{~F}(4.077,195.709)=1.006, \mathrm{p}=0.406)$. These results show that the fish appeared to habituate

278 after introduction to the novel tank, as the total distance per minute and mean ambulatory speed

279 gradually increased across the duration of the novel tank test, but there was no effect of treatment

280 on these measures.

281 Freezing behavior in the novel tank test

282

A t-test for independent means indicated no significant effect of chronic unpredictable

283 stress on the total immobility time $(\mathrm{t}(48)=0.544, \mathrm{p}=0.589)$ in the novel tank test (Table 2$)$.

284 When the immobility data was broken down into six 60-second bins (Figure 2) and analyzed

285 with a repeated-measures ANOVA, there was no effect of stress $(F(1,48)=0.359, p=0.552)$, no

286 effect of time $(F(2.191,105.178)=1.359, \mathrm{p}=0.262)$, and no interaction between stress and time

$287(\mathrm{~F}(2.191,105.178)=1.114, \mathrm{p}=0.336)$. Thus, neither time nor treatment significantly altered

288 freezing behavior across the six minutes of the novel tank test.

289 Exploratory behavior in the novel tank test

290 A t-test for independent means indicated a marginally significant effect of chronic

291 unpredictable stress for the number of entries to the top zone $(\mathrm{t}(48)=-1.920, \mathrm{p}=0.061$; CUS $<$

292 untreated), a significant effect on the total time spent in the top zone $(\mathrm{t}(48)=-2.129, \mathrm{p}=0.038$;

293 CUS $<$ untreated), but no significant difference in the distance traveled in the top zone $(t(48)=$ -

$2941.309, \mathrm{p}=0.197$; Table 2). When the number of entries to the top zone was broken down into six

295 60-second bins (Figure 3A) and analyzed with a repeated-measures ANOVA, there was a 
296 marginal effect of stress $(F(1,48)=3.454, p=0.069)$, a significant effect of time

$297(\mathrm{~F}(3.855,185.031)=6.701, \mathrm{p}<0.001)$, but no interaction between stress and time

$298(\mathrm{~F}(3.855,185.031)=0.935, \mathrm{p}=0.442)$. For the time spent in the top zone (Figure 3B), there was

299 a significant effect of stress $(F(1,48)=4.530, p=0.038)$, a significant effect of time

$300 \quad(\mathrm{~F}(3.540,169.936)=13.317, \mathrm{p}<0.001)$, but no interaction between stress and time

$301(\mathrm{~F}(3.540,169.936)=1.318, \mathrm{p}=0.268)$. For the distance traveled in the top zone (Figure $3 \mathrm{C})$,

302 there was no effect of stress $(F(1,48)=1.635, p=0.207)$, a significant effect of time

$303(\mathrm{~F}(3.631,174.291)=12.430, \mathrm{p}<0.001)$, but no interaction between stress and time

$304(\mathrm{~F}(3.631,174.291)=1.408, \mathrm{p}=0.237)$. Similar to the motor measures, the fish appeared to

305 habituate and explore more of the top zone of the novel tank over time, but the fish that were

306 chronically stressed generally explored the top zone less than the fish that were left untreated for 307 seven days.

\section{Trunk cortisol}

309 A t-test for independent means indicated that fish exposed to seven days of chronic

310 unpredictable stress (CUS) had decreased basal levels of trunk cortisol compared to untreated

311 control fish $(\mathrm{t}(48)=-3.130, \mathrm{p}=0.003$; Figure $4 \mathrm{~A})$. This finding suggests that seven days of

312 chronic unpredictable stress decreases basal levels of cortisol in zebrafish compared to fish that

313 were untreated.

\section{Trunk weights}

315 A t-test for independent means indicated that fish exposed to 7 days of chronic

316 unpredictable stress (CUS) had similar trunk weights as fish that were left untreated for 7 days

$317(\mathrm{t}(48)=-1.111, \mathrm{p}=0.272$; Figure 4B). This finding suggests that 7 days of chronic unpredictable 
318 stress does not appear to alter factors involved with body weight regulation, such as feeding, in

319 zebrafish.

320 Experiment 2: Effects of seven days of chronic unpredictable stress (CUS) treatment and seven

321 days of prazosin treatment on behavioral measures in the novel tank test (NTT), basal levels of

322 cortisol, and body weights in adult zebrafish.

323 Motor activity in the novel tank test

324 A two-way ANOVA indicated that there was no effect of stress $(\mathrm{F}(1,65)=0.358, \mathrm{p}=$

$3250.552)$, no effect of drug treatment $(F(1,65)=0.139, p=0.710)$, and no interaction between

326 stress and drug $(\mathrm{F}(1,65)=0.103, \mathrm{p}=0.750)$ on the total distance traveled in the novel tank test

327 (Table 3). A two-way ANOVA indicated that there was no effect of stress $(F(1,65)=0.657, \mathrm{p}=$

$3280.421)$, no effect of drug treatment $(F(1,65)=1.186, p=0.280)$, and no interaction between

329 stress and drug $(\mathrm{F}(1,65)=0.355, \mathrm{p}=0.553)$ on the mean ambulatory speed of fish in the novel

330 tank test (Table 3). The total distance (Figure 5A) and mean ambulatory speed (Figure 5B) data

331 was also broken down into six 60-second bins and analyzed with a repeated-measures ANOVA

332 (see Table 4 for statistical analyses). These results indicate that, similar to the results from

333 Experiment 1, fish appear to habituate to the novel tank across the duration of the test, but that

334 there is no effect of stress or drug treatment on these measures of motor activity.

335 Freezing behavior in the novel tank test

336 A two-way ANOVA indicated that there was a marginal effect of stress $(\mathrm{F}(1,65)=3.050$,

$337 \mathrm{p}=0.085 ;$ CUS $<$ control $)$, a marginal effect of drug treatment $(\mathrm{F}(1,65)=3.050, \mathrm{p}=0.085$;

338 prazosin $<$ vehicle), but no interaction between stress and drug $(\mathrm{F}(1,65)=2.087, \mathrm{p}=0.153)$ on

339 total immobility time in the novel tank test (Table 3). The immobility data (Figure 6) was also

340 broken down into six 60-second bins and analyzed with a repeated-measures ANOVA (see Table 
3414 for statistical analyses). These results indicate that immobility generally decreases across the

342 duration of the novel tank test, but that there is no significant effect of stress or drug treatment on

343 this behavioral measure.

344 Exploratory behavior in the novel tank test

A two-way ANOVA indicated that there was a significant effect of stress $(\mathrm{F}(1,65)=$

$5.939, \mathrm{p}=0.018$; CUS $>$ control $)$, a marginally significant effect of drug treatment $(\mathrm{F}(1,65)=$

2.903, $\mathrm{p}=0.093$; prazosin $>$ vehicle $)$, but no interaction between stress and $\operatorname{drug}(\mathrm{F}(1,65)=$

$1.404, p=0.240$ ) on the number of entries to the top zone (Table 3). A two-way ANOVA

indicated that there was no effect of stress $(F(1,65)=2.769, p=0.101)$, a significant effect of

drug treatment $(\mathrm{F}(1,65)=4.061, \mathrm{p}=0.048$; prazosin $>$ vehicle $)$, but no interaction between stress and drug $(\mathrm{F}(1,65)=0.956, \mathrm{p}=0.332)$ on the total amount of time spent in the novel tank test

352 (Table 3). A two-way ANOVA indicated that there was a significant effect of stress $(\mathrm{F}(1,65)=$ $4.876, p=0.031$; CUS $>$ control $)$, no effect of drug treatment $(F(1,65)=0.745, p=0.391)$, but no interaction between stress and drug $(\mathrm{F}(1,65)=1.947, \mathrm{p}=0.168)$ on the distance traveled in the top zone of the novel tank (Table 3). The number of entries to the top (Figure 7A), time spent in the top zone (Figure 7B), and distance traveled in the top zone (Figure 7C) were also broken down into six 60-second bins and analyzed with a repeated-measures ANOVA (see Table 4 for statistical analyses). These results suggest that allowing additional time (seven days) to elapse between the chronic stress paradigm and testing in the novel tank perhaps reverses the deficits in top zone exploration elicited by CUS observed in Experiment 1. In addition, these results suggest that chronic prazosin increases exploration in the novel tank test in the absence of stress, but

362 does not appear to alter any stress-induced effects on top zone exploration in the novel tank when 363 prazosin treatment follows chronic unpredictable stress. 


\section{Trunk cortisol}

365

366

367

368

369

370

371

372

373

374

375

376

377

378

379

380

381

382

383

384

385

386

A two-way ANOVA indicated that there was no effect of stress $(F(1,65)=2.511, \mathrm{p}=$ $0.118)$, no effect of drug treatment $(F(1,65)=0.624, p=0.432)$, and no interaction between stress and drug $(\mathrm{F}(1,65)=0.636, \mathrm{p}=0.428)$ on basal levels of cortisol (Figure 8A). Although these results did not reach statistical significance, the fish subjected to chronic unpredictable stress and subsequently were vehicle-treated had lower levels of cortisol than untreated-vehicle controls, which is similar to the pattern of results observed in Experiment 1. Prazosin treated-fish also had lower levels of basal cortisol compared to unstressed/vehicle-treated fish; however, this finding did not reach statistical significance.

\section{Trunk weights}

A two-way ANOVA indicated that there was no effect of stress $(F(1,65)=0.057, p=$ $0.811)$, no effect of drug treatment $(F(1,65)=0.173, p=0.679)$, and no interaction between stress and drug $(\mathrm{F}(1,65)=0.467, \mathrm{p}=0.497)$ on subject trunk weights (Figure $8 \mathrm{~B})$. Similar to the results from Experiment 1, these results indicate that neither stress treatment nor chronic drug treatment altered factors involved with body weight regulation.

9

\section{Discussion}

The noradrenergic system is critical for the regulation of several functions, including the regulation of stress responses. The locus coeruleus, the major noradrenergic nucleus of the brain, supplies norepinephrine both systemically and directly to regions throughout the brain including the amygdala, hypothalamus, and the medial prefrontal cortex (mPFC), all areas involved with regulating responses to stress. Noradrenergic dysfunction has been hypothesized to be involved with the neuropathology associated with PTSD (Hendrickson \& Raskind, 2016; O'Donnell, 
387 Hegadoren, \& Coupland, 2004; Southwick, Bremner, et al., 1999; Southwick, Paige, et al., 1999;

388 Strawn \& Geracioti, 2008). Dysregulation of the noradrenergic system may ultimately contribute

389 to the alterations in the function of the physiological stress axis observed in patients and animal

390 models. Thus, pharmacological agents that target the norepinephrine regulation of stress

391 responses have the potential to normalize neuroendocrine dysfunction associated with stress-

392 related conditions.

393 The hypothesis of the current study was that the zebrafish model of CUS would increase

394 basal levels of cortisol and increase anxiety-like behavior in the novel tank test, and that chronic

395 prazosin treatment would reverse alterations induced by chronic stress. In support of this

396 hypothesis, CUS slightly increased anxiety-like behavior in the novel tank test; however, this

397 change in behavior was associated with significantly lower levels of basal cortisol. In addition,

398 prazosin appeared to decrease levels of anxiety-like behavior in the absence of CUS. However, it

399 also appeared that CUS-induced decreases in exploratory behavior and basal cortisol levels

400 started to normalize (and in the case of the behavioral variables, reversed) in the $7 \mathrm{~d}$ interim when

401 vehicle and drug treatment were being administered between CUS and dependent variable

402 assessments.

403 The study design and timing of the assessment of the dependent variables should be

404 considered when interpreting the results of the current study. In the first experiment, behavioral

405 and neuroendocrine measurements were assessed immediately after the seven days of chronic

406 stress. In the second experiment, subjects were exposed to seven days of chronic stress followed

407 by a week of chronic drug treatment administered in the absence of unpredictable stressors. It is

408 possible that any stress-induced effects may have been blunted or reversed by the time the

409 measurements were assessed. For example, in Experiment 1, fish that were exposed to CUS had 
410 lower levels of basal cortisol compared to unstressed fish. In Experiment 2, fish that were

411 chronically stressed but subsequently treated with vehicle for seven days still had lower basal

412 cortisol levels than non-stressed, vehicle treated subjects; however, this difference did not reach

413 the criterion for statistical significance (see Figure 8A). Alternatively, in Experiment 2, the

414 additional week of handling necessary to administer the drug/vehicle treatment may have

415 triggered adaptive mechanisms in animals previously exposed to the CUS paradigm, which could

416 have increased the elevated exploratory behavior observed in the novel tank test compared to

417 non-stressed, vehicle-treated controls (see Figure 7). Baseline measures of anxiety could have

418 been increased with the 7 additional days of handling; this supposition is supported by the fact

419 that unstressed/vehicle-treated fish in Experiment 2 generally displayed more anxiety-like

420 behavior (increased immobility and decreased top zone exploration) compared to the unstressed

421 fish from Experiment 1 (see group means in Tables 2 and 3). Future studies should address the

422 duration or persistence of long-term neuroendocrine and behavioral effects of 7d CUS and

423 whether chronic prazosin treatment administered during the same period as the stressors would

424 circumvent any possible effects of allowing previously stressed animals to adapt back to non-

425 stressed conditions. Other studies have examined the effects of anxiolytic compounds

426 administered concurrent with chronic stress paradigms; for example, in a previous study,

427 zebrafish were exposed to chronic unpredictable stress for five weeks but then were treated with

428 the antidepressant fluoxetine during the last 8 days of the stressor paradigm (Song et al., 2018).

429 Another study exposed zebrafish to chronic unpredictable stress for 14 days but treated with the

430 putative anxiolytic $\mathrm{N}$-acetylcysteine concurrent with the last 7 days of the stress (Mocelin et al.,

431 2019). Thus, it would be interesting to see whether continuing the chronic unpredictable stress 
432 exposure during treatment or by conducting the stress exposure during the dark phase (Manuel et

433 al., 2014) would make prazosin-induced effects more obvious.

434 In the current report, prazosin treatment alone appeared to enhance exploratory behavior

435 in the novel tank test but did not affect CUS-induced changes in anxiety-like behavior. In

436 addition, prazosin-treated groups had lower levels of cortisol than non-stressed, vehicle-treated

437 subjects, although this decrease did not reach statistical significance. Prazosin treatment may

438 function to generally prevent reactions to stress (Rasmussen, Kincaid, \& Froehlich, 2017); thus,

439 further studies should also determine whether preventative prazosin treatment is effective at

440 blocking any chronic or acute stress effects on anxiety-like behavior or neuroendocrine

441 dysfunction. These studies would help clarify whether prazosin would be clinically efficacious,

442 not by normalizing the effects of trauma or stress, but by preventing any responses to further

443 triggering stimuli that may elicit PTSD symptomology. As prazosin acts by putatively blocking

$444 \alpha 1$ receptors, antagonism of alpha-1 receptors could possibly preventing further stress or trauma

445 from triggering the norepinephrine-mediated stimulation of stress axis reactivity (Ma \& Morilak,

446 2005). Thus, prazosin may work better as a prophylactic in treating stress-related conditions, as

447 has been observed for other medications (Roque, 2015), although much more clinical work

448 would be necessary to establish this as a potential option for therapy.

449 It is also interesting to note that, although it was expected that seven days of CUS

450 treatment would elicit increases in basal cortisol levels based on previously published reports

451 (Manuel et al., 2014; Marcon et al., 2016, 2018; Piato et al., 2011), the results from the first

452 experiment indicate that the CUS protocol can elicit hypocortisolic responses. There are several

453 factors that could explain the different results between laboratories, such as the source, strain,

454 previous stress exposure, or age of the subjects. For example, one previous report indicated that 
455 there are possible sex-specific differences in basal cortisol levels after exposure to chronic stress, 456 with male zebrafish exhibiting increases in cortisol compared to untreated controls, while the

457 levels of basal cortisol did not change in females relative to untreated controls (Rambo et al., 458 2017). Thus, the impact of both prazosin and chronic stress on our dependent measures may be 459 masked by including both sexes in the analyses. The current results support a recent call for 460 much more research into the housing, breeding, and other husbandry conditions that may be 461 contributing factors to differences in experimental results between laboratories (Lidster,

462 Readman, Prescott, \& Owen, 2017; Tsang et al., 2017; Varga, Ekker, \& Lawrence, 2018).

463 Another factor that could play a role in our findings is the binding profile of prazosin to $464 \alpha 1$ adrenergic receptors in the zebrafish brain. Although the binding of prazosin in brain has been 465 extensively studied in rodent and other mammalian models (Dashwood, 1982; Greengrass \& 466 Bremner, 1979; Mignot, Bowersox, Maddaluno, Dement, \& Ciaranello, 1989; Morrow,

467 Battaglia, Norman, \& Creese, 1985; Morrow \& Creese, 1986; Rainbow \& Biegon, 1983), the 468 same cannot be said about prazosin binding in zebrafish brain. Studies using preparations of 469 codfish brain indicate that prazosin binding may in fact be different in fish brains compared to 470 rodent brains (Bergström \& Wikberg, 1986a, 1986b). Perhaps more importantly, although 471 zebrafish $\alpha 2$ and $\beta$ receptor binding and distribution has been explored (Ampatzis \& Dermon, 472 2010; Ampatzis, Kentouri, \& Dermon, 2008; Ruuskanen, Laurila, et al., 2005; Ruuskanen, 473 Peitsaro, Kaslin, Panula, \& Scheinin, 2005; Wang et al., 2009), little comparable information on 474 zebrafish $\alpha 1$ receptor binding and distribution is available in the extant literature. Thus, more 475 work is necessary for a complete understanding of noradrenergic modulation of stress responses 476 in the zebrafish model. 

stress-related conditions like PTSD does not involve the normalization of physiological stress axis dysfunction. Rather, the clinical effectiveness of prazosin likely involves other mechanisms of altering stress regulation. In addition, this study also highlights the importance of considering studies, which will ultimately contribute to a better understanding of the complex nature of the regulation and expression of stress responses.

\section{Conclusions}

This study demonstrated that seven days of chronic unpredictable stress exposure in zebrafish increased the expression of anxiety-like behavior and decreased basal levels of cortisol. When prazosin, a putative alpha-1 receptor antagonist, was chronically administered to subjects after the stress exposure, stress-related effects on behavior and hormones were not reversed; however, prazosin appeared to decrease anxiety-like behaviors in the novel tank test in the absence of stress exposure. Further studies are necessary to determine the longevity of chronic stress-induced responses and whether effects on stress-induced alterations in stress responses are dependent on the timing of drug treatment. These studies also suggest that normalization of neuroendocrine dysfunction may not be involved with the clinical efficacy of prazosin in the treatment of PTSD, although human studies are needed to confirm this finding. 


\section{Acknowledgements}

501 The authors would like to thank Dr. Harold Olivey and Dr. Jenny Fisher of the Indiana

502 University Northwest Department of Biology for their technical assistance with some aspects of 503 these studies.

504

505

506

507

508

509

510

511

512

513

514

515

516

517

518

519

520

521

522

Peer) reviewing PDF | (2019:04:36970:1:1:NEW 4 Dec 2019) 
524 Ahmadpanah, M., Sabzeiee, P., Hosseini, S. M., Torabian, S., Haghighi, M., Jahangard, L., ...

525 Brand, S. (2014). Comparing the effect of prazosin and hydroxyzine on sleep quality in 526 patients suffering from posttraumatic stress disorder. Neuropsychobiology, 69(4), 235242. https://doi.org/10.1159/000362243

Alia, A. O., \& Petrunich-Rutherford, M. L. (2019). Anxiety-like behavior and whole-body cortisol responses to components of energy drinks in zebrafish (Danio rerio). PeerJ, 7, e7546. https://doi.org/10.7717/peerj.7546

Ampatzis, K., \& Dermon, C. R. (2010). Regional distribution and cellular localization of beta2adrenoceptors in the adult zebrafish brain (Danio rerio). The Journal of Comparative Neurology, 518(9), 1418-1441. https://doi.org/10.1002/cne.22278

Ampatzis, K., Kentouri, M., \& Dermon, C. R. (2008). Neuronal and glial localization of alpha(2A)-adrenoceptors in the adult zebrafish (Danio rerio) brain. The Journal of Comparative Neurology, 508(1), 72-93. https://doi.org/10.1002/cne.21663

Aponte, A., \& Petrunich-Rutherford, M. L. (2019). Acute net stress of young adult zebrafish (Danio rerio) is not sufficient to increase anxiety-like behavior and whole-body cortisol. PeerJ, 7, e7469. https://doi.org/10.7717/peerj.7469

Bergström, A., \& Wikberg, J. E. (1986a). Guanine nucleotides regulate both agonist and antagonist binding to cod brain alpha 1-adrenoceptors. Acta Pharmacologica Et Toxicologica, 59(4), 270-278. https://doi.org/10.1111/j.1600-0773.1986.tb00168.x

Bergström, A., \& Wikberg, J. E. (1986b). Structural and pharmacological differences between codfish and rat brain alpha 1-adrenergic receptors revealed by photoaffinity labeling with 125I-APDQ. Acta Pharmacologica Et Toxicologica, 58(2), 148-155. 
https://doi.org/10.1111/j.1600-0773.1986.tb00085.x

547 Bisson, J. I., Cosgrove, S., Lewis, C., \& Robert, N. P. (2015). Post-traumatic stress disorder. BMJ (Clinical Research Ed.), 351, h6161. https://doi.org/10.1136/bmj.h6161

Cachat, J., Stewart, A., Grossman, L., Gaikwad, S., Kadri, F., Chung, K. M., ... Kalueff, A. V. (2010). Measuring behavioral and endocrine responses to novelty stress in adult zebrafish. Nature Protocols, 5(11), 1786-1799. https://doi.org/10.1038/nprot.2010.140

Canavello, P. R., Cachat, J. M., Beeson, E. C., Laffoon, A. L., Grimes, C., Haymore, W. A. M., ... Kalueff, A. V. (2011). Measuring Endocrine (Cortisol) Responses of Zebrafish to Stress. In Neuromethods: Vol. 51. Zebrafish Neurobehavioral Protocols (pp. 135-142). New York, NY: Humana Press.

Caramillo, E. M., Khan, K. M., Collier, A. D., \& Echevarria, D. J. (2015). Modeling PTSD in the zebrafish: Are we there yet? Behavioural Brain Research, 276, 151-160.

Chakravarty, S., Reddy, B. R., Sudhakar, S. R., Saxena, S., Das, T., Meghah, V., ... Idris, M. M. (2013). Chronic unpredictable stress (CUS)-induced anxiety and related mood disorders in a zebrafish model: Altered brain proteome profile implicates mitochondrial

Clark, K. J., Boczek, N. J., \& Ekker, S. C. (2011). Stressing zebrafish for behavioral genetics. dysfunction. PloS One, 8(5), e63302. https://doi.org/10.1371/journal.pone.0063302 Reviews in the Neurosciences, 22(1), 49-62. https://doi.org/10.1515/RNS.2011.007 study in the rat. European Journal of Pharmacology, 86(1), 51-58. https://doi.org/10.1016/0014-2999(82)90395-8

Davis, D. J., Klug, J., Hankins, M., Doerr, H. M., Monticelli, S. R., Song, A., ... Bryda, E. C. 
(2015). Effects of Clove Oil as a Euthanasia Agent on Blood Collection Efficiency and Serum Cortisol Levels in Danio rerio. Journal of the American Association for Laboratory Animal Science: JAALAS, 54(5), 564-567.

572

De Berardis, D., Marini, S., Serroni, N., Iasevoli, F., Tomasetti, C., de Bartolomeis, A., ... Di Giannantonio, M. (2015). Targeting the Noradrenergic System in Posttraumatic Stress Disorder: A Systematic Review and Meta-Analysis of Prazosin Trials. Current Drug Targets, 16(10), 1094-1106.

Deka, R., Bryan, C. J., LaFleur, J., Oderda, G., Atherton, A., \& Stevens, V. (2018). Benzodiazepines, Health Care Utilization, and Suicidal Behavior in Veterans With Posttraumatic Stress Disorder. The Journal of Clinical Psychiatry, 79(6). https://doi.org/10.4088/JCP.17m12038

Dhanasiri, A. K. S., Fernandes, J. M. O., \& Kiron, V. (2013). Acclimation of zebrafish to transport stress. Zebrafish, 10(1), 87-98. https://doi.org/10.1089/zeb.2012.0843

Dunlop, B. W., \& Wong, A. (2019). The hypothalamic-pituitary-adrenal axis in PTSD: Pathophysiology and treatment interventions. Progress in Neuro-Psychopharmacology \& Biological Psychiatry, 89, 361-379. https://doi.org/10.1016/j.pnpbp.2018.10.010

Egan, R. J., Bergner, C. L., Hart, P. C., Cachat, J. M., Canavello, P. R., Elegante, M. F., ... Kalueff, A. V. (2009). Understanding behavioral and physiological phenotypes of stress and anxiety in zebrafish. Behavioural Brain Research, 205(1), 38-44. https://doi.org/10.1016/j.bbr.2009.06.022

Faul, F., Erdfelder, E., Lang, A.-G., \& Buchner, A. (2007). G*Power 3: A flexible statistical power analysis program for the social, behavioral, and biomedical sciences. Behavior Research Methods, 39(2), 175-191. https://doi.org/10.3758/bf03193146 
592 Foa, E. B., Keane, T. M., Friedman, M. J., \& Cohen, J. A. (2009). Effective treatments for PTSD:

593 practice guidelines from the International Society for Traumatic Stress Studies (2nd ed.).

$594 \quad$ New York, NY: The Guilford Press.

595 Fox, H. C., Anderson, G. M., Tuit, K., Hansen, J., Kimmerling, A., Siedlarz, K. M., ... Sinha, R.

596 (2012). Prazosin effects on stress- and cue-induced craving and stress response in

597

598 alcohol-dependent individuals: Preliminary findings. Alcoholism, Clinical and Experimental Research, 36(2), 351-360. https://doi.org/10.1111/j.1530-

599 0277.2011.01628.x

600 Fulcher, N., Tran, S., Shams, S., Chatterjee, D., \& Gerlai, R. (2017). Neurochemical and 601 602 603 Behavioral Responses to Unpredictable Chronic Mild Stress Following Developmental Isolation: The Zebrafish as a Model for Major Depression. Zebrafish, 14(1), 23-34. https://doi.org/10.1089/zeb.2016.1295

Green, B. (2014). Prazosin in the treatment of PTSD. Journal of Psychiatric Practice, 20(4), 253-259. https://doi.org/10.1097/01.pra.0000452561.98286.1e

Greengrass, P., \& Bremner, R. (1979). Binding characteristics of 3H-prazosin to rat brain alphaadrenergic receptors. European Journal of Pharmacology, 55(3), 323-326. https://doi.org/10.1016/0014-2999(79)90202-4

610 Hendrickson, R. C., \& Raskind, M. A. (2016). Noradrenergic dysregulation in the pathophysiology of PTSD. Experimental Neurology, 284(Pt B), 181-195.

613 Kessler, R. C., Aguilar-Gaxiola, S., Alonso, J., Benjet, C., Bromet, E. J., Cardoso, G., ... Koenen, K. C. (2017). Trauma and PTSD in the WHO World Mental Health Surveys. 
615

616

617 Koenen, K. C., Ratanatharathorn, A., Ng, L., McLaughlin, K. A., Bromet, E. J., Stein, D. J., ...

618 Kessler, R. C. (2017). Posttraumatic stress disorder in the World Mental Health Surveys.

$619 \quad$ Psychological Medicine, 47(13), 2260-2274.

620

621

622

623

624

625

626

627

628

629

630

631

632

633

634

635

636

637
European Journal of Psychotraumatology, 8(sup5), 1353383.

https://doi.org/10.1080/20008198.2017.1353383
Koola, M. M., Varghese, S. P., \& Fawcett, J. A. (2014). High-dose prazosin for the treatment of post-traumatic stress disorder. Therapeutic Advances in Psychopharmacology, 4(1), 4347. https://doi.org/10.1177/2045125313500982

Lancaster, C. L., Teeters, J. B., Gros, D. F., \& Back, S. E. (2016). Posttraumatic Stress Disorder: Overview of Evidence-Based Assessment and Treatment. Journal of Clinical Medicine, 5(11). https://doi.org/10.3390/jcm5110105

Lidster, K., Readman, G. D., Prescott, M. J., \& Owen, S. F. (2017). International survey on the use and welfare of zebrafish Danio rerio in research. Journal of Fish Biology, 90(5), 1891-1905. https://doi.org/10.1111/jfb.13278

Loeffler, G., Coller, R., Tracy, L., \& Derderian, B. R. (2018). Prescribing Trends in US Active Duty Service Members With Posttraumatic Stress Disorder: A Population-Based Study From 2007-2013. The Journal of Clinical Psychiatry, 79(4). https://doi.org/10.4088/JCP.17m11667

Ma, S., \& Morilak, D. A. (2005). Norepinephrine release in medial amygdala facilitates activation of the hypothalamic-pituitary-adrenal axis in response to acute immobilisation stress. Journal of Neuroendocrinology, 17(1), 22-28. https://doi.org/10.1111/j.13652826.2005.01279.x 
638 Manuel, R., Gorissen, M., Zethof, J., Ebbesson, L. O. E., van de Vis, H., Flik, G., \& van den 639 Bos, R. (2014). Unpredictable chronic stress decreases inhibitory avoidance learning in 640 Tuebingen long-fin zebrafish: Stronger effects in the resting phase than in the active 641 phase. The Journal of Experimental Biology, 217(Pt 21), 3919-3928.

642 https://doi.org/10.1242/jeb.109736

643

644

645

646

647

648

649

650

651

652

653

654

655

656

657

658

659

660

Marcon, M., Herrmann, A. P., Mocelin, R., Rambo, C. L., Koakoski, G., Abreu, M. S., ... Piato, A. L. (2016). Prevention of unpredictable chronic stress-related phenomena in zebrafish exposed to bromazepam, fluoxetine and nortriptyline. Psychopharmacology, 233(21-22), 3815-3824. https://doi.org/10.1007/s00213-016-4408-5

Marcon, M., Mocelin, R., Benvenutti, R., Costa, T., Herrmann, A. P., de Oliveira, D. L., ... Piato, A. (2018). Environmental enrichment modulates the response to chronic stress in zebrafish. The Journal of Experimental Biology, 221(Pt 4). https://doi.org/10.1242/jeb.176735

Mason, J. W., Giller, E. L., Kosten, T. R., Ostroff, R. B., \& Podd, L. (1986). Urinary freecortisol levels in posttraumatic stress disorder patients. The Journal of Nervous and Mental Disease, 174(3), 145-149. https://doi.org/10.1097/00005053-198603000-00003

Mignot, E., Bowersox, S. S., Maddaluno, J., Dement, W., \& Ciaranello, R. (1989). Evidence for multiple $[3 \mathrm{H}]$ prazosin binding sites in canine brain membranes. Brain Research, 486(1), 56-66. https://doi.org/10.1016/0006-8993(89)91277-8

Mocelin, R., Marcon, M., D’ambros, S., Mattos, J., Sachett, A., Siebel, A. M., ... Piato, A. (2019). N-Acetylcysteine Reverses Anxiety and Oxidative Damage Induced by Unpredictable Chronic Stress in Zebrafish. Molecular Neurobiology, 56(2), 1188-1195. https://doi.org/10.1007/s12035-018-1165-y 
661 Morrow, A. L., Battaglia, G., Norman, A. B., \& Creese, I. (1985). Identification of subtypes of $662[3 \mathrm{H}]$ prazosin-labelled alpha 1 receptor binding sites in rat brain. European Journal of 663 Pharmacology, 109(2), 285-287. https://doi.org/10.1016/0014-2999(85)90432-7

664 Morrow, A. L., \& Creese, I. (1986). Characterization of alpha 1-adrenergic receptor subtypes in 665 rat brain: A reevaluation of [3H]WB4104 and [3H]prazosin binding. Molecular

666 Pharmacology, 29(4), 321-330.

667 National Research Council. (2011). Guide for the Care and Use of Laboratory Animals (8th ed.). 668 Washington DC: The National Academies Press.

669 Nesan, D., \& Vijayan, M. M. (2013). Role of glucocorticoid in developmental programming:

670 Evidence from zebrafish. General and Comparative Endocrinology, 181, 35-44.

671 https://doi.org/10.1016/j.ygcen.2012.10.006

672 O’Donnell, T., Hegadoren, K. M., \& Coupland, N. C. (2004). Noradrenergic mechanisms in the 673 pathophysiology of post-traumatic stress disorder. Neuropsychobiology, 50(4), 273-283. https://doi.org/10.1159/000080952

Papapetropoulos, A., \& Szabo, C. (2018). Inventing new therapies without reinventing the wheel: The power of drug repurposing. British Journal of Pharmacology, 175(2), 165167. https://doi.org/10.1111/bph.14081 Progress in Brain Research, 182, 149-160. https://doi.org/10.1016/S0079-

680 6123(10)82005-9

681 Pétursson, H. (1994). The benzodiazepine withdrawal syndrome. Addiction (Abingdon, 682 England), 89(11), 1455-1459.

683 Piato, Â. L., Capiotti, K. M., Tamborski, A. R., Oses, J. P., Barcellos, L. J. G., Bogo, M. R., ... 
684

685

686

687

688

689

690

691

692

693

694

695

696

697

698

699

700

701

702

703

704

705

706

Bonan, C. D. (2011). Unpredictable chronic stress model in zebrafish (Danio rerio): Behavioral and physiological responses. Progress in Neuro-Psychopharmacology \& Biological Psychiatry, 35(2), 561-567. https://doi.org/10.1016/j.pnpbp.2010.12.018

Pilehvar, A., Town, R. M., \& Blust, R. (2020). The effect of copper on behaviour, memory, and associative learning ability of zebrafish (Danio rerio). Ecotoxicology and Environmental Safety, 188, 109900. https://doi.org/10.1016/j.ecoenv.2019.109900

Rainbow, T. C., \& Biegon, A. (1983). Quantitative autoradiography of [3H]prazosin binding sites in rat forebrain. Neuroscience Letters, 40(3), 221-226. https://doi.org/10.1016/03043940(83)90042-3

Rambo, C. L., Mocelin, R., Marcon, M., Villanova, D., Koakoski, G., de Abreu, M. S., ... Bonan, C. D. (2017). Gender differences in aggression and cortisol levels in zebrafish subjected to unpredictable chronic stress. Physiology \& Behavior, 171, 50-54. https://doi.org/10.1016/j.physbeh.2016.12.032

Rasmussen, D. D., Kincaid, C. L., \& Froehlich, J. C. (2017). Prazosin Prevents Increased Anxiety Behavior That Occurs in Response to Stress During Alcohol Deprivations. Alcohol and Alcoholism (Oxford, Oxfordshire), 52(1), 5-11. https://doi.org/10.1093/alcalc/agw082

Roque, A. P. (2015). Pharmacotherapy as prophylactic treatment of post-traumatic stress disorder: A review of the literature. Issues in Mental Health Nursing, 36(9), 740-751. https://doi.org/10.3109/01612840.2015.1057785

Ruuskanen, J. O., Laurila, J., Xhaard, H., Rantanen, V.-V., Vuoriluoto, K., Wurster, S., ... Scheinin, M. (2005). Conserved structural, pharmacological and functional properties among the three human and five zebrafish alpha 2-adrenoceptors. British Journal of 
708

709

710

711

712

713

714

715

716

717

718

719

720

721

722

723

724

725

726

727

728

729

Ruuskanen, J. O., Peitsaro, N., Kaslin, J. V. M., Panula, P., \& Scheinin, M. (2005). Expression and function of alpha-adrenoceptors in zebrafish: Drug effects, mRNA and receptor distributions. Journal of Neurochemistry, 94(6), 1559-1569. https://doi.org/10.1111/j.1471-4159.2005.03305.x

Simon, P. Y. R., \& Rousseau, P.-F. (2017). Treatment of Post-Traumatic Stress Disorders with the Alpha-1 Adrenergic Antagonist Prazosin. Canadian Journal of Psychiatry. Revue Canadienne De Psychiatrie, 62(3), 186-198. https://doi.org/10.1177/0706743716659275

Singh, A., Subhashini, N., Sharma, S., \& Mallick, B. N. (2013). Involvement of the $\alpha 1-$ adrenoceptor in sleep-waking and sleep loss-induced anxiety behavior in zebrafish. Neuroscience, 245, 136-147. https://doi.org/10.1016/j.neuroscience.2013.04.026

Singh, B., Hughes, A. J., Mehta, G., Erwin, P. J., \& Parsaik, A. K. (2016). Efficacy of Prazosin in Posttraumatic Stress Disorder: A Systematic Review and Meta-Analysis. The Primary Care Companion for CNS Disorders, 18(4). https://doi.org/10.4088/PCC.16r01943

Song, C., Liu, B.-P., Zhang, Y.-P., Peng, Z., Wang, J., Collier, A. D., ... Kalueff, A. V. (2018). Modeling consequences of prolonged strong unpredictable stress in zebrafish: Complex effects on behavior and physiology. Progress in Neuro-Psychopharmacology \& Biological Psychiatry, 81, 384-394. https://doi.org/10.1016/j.pnpbp.2017.08.021

Southwick, S. M., Bremner, J. D., Rasmusson, A., Morgan, C. A., Arnsten, A., \& Charney, D. S. (1999). Role of norepinephrine in the pathophysiology and treatment of posttraumatic stress disorder. Biological Psychiatry, 46(9), 1192-1204. https://doi.org/10.1016/s00063223(99)00219-x

Southwick, S. M., Paige, S., Morgan, C. A., Bremner, J. D., Krystal, J. H., \& Charney, D. S. 
732 Stewart, A. M., Yang, E., Nguyen, M., \& Kalueff, A. V. (2014). Developing zebrafish models relevant to PTSD and other trauma- and stressor-related disorders. Progress in NeuroPsychopharmacology \& Biological Psychiatry, 55, 67-79. https://doi.org/10.1016/j.pnpbp.2014.08.003

736

Strawn, J. R., \& Geracioti, T. D. (2008). Noradrenergic dysfunction and the psychopharmacology of posttraumatic stress disorder. Depression and Anxiety, 25(3), 260-271. https://doi.org/10.1002/da.20292

Tsang, B., Zahid, H., Ansari, R., Lee, R. C.-Y., Partap, A., \& Gerlai, R. (2017). Breeding Zebrafish: A Review of Different Methods and a Discussion on Standardization. Zebrafish, 14(6), 561-573. https://doi.org/10.1089/zeb.2017.1477

Varga, Z. M., Ekker, S. C., \& Lawrence, C. (2018). Workshop Report: Zebrafish and Other Fish Models-Description of Extrinsic Environmental Factors for Rigorous Experiments and Reproducible Results. Zebrafish, 15(6), 533-535. https://doi.org/10.1089/zeb.2018.29006.zol

Wang, Z., Nishimura, Y., Shimada, Y., Umemoto, N., Hirano, M., Zang, L., ... Tanaka, T. (2009). Zebrafish beta-adrenergic receptor mRNA expression and control of pigmentation. Gene, 446(1), 18-27. https://doi.org/10.1016/j.gene.2009.06.005

Wendelaar Bonga, S. E. (1997). The stress response in fish. Physiological Reviews, 77(3), 591625. https://doi.org/10.1152/physrev.1997.77.3.591

Westerfield, M. (2000). The zebrafish book: A guide for the laboratory use of zebrafish (Danio rerio) (4th ed.). Eugene: University of Oregon Press. 
753 Wichmann, S., Kirschbaum, C., Böhme, C., \& Petrowski, K. (2017). Cortisol stress response in 754 post-traumatic stress disorder, panic disorder, and major depressive disorder patients. Psychoneuroendocrinology, 83, 135-141. https://doi.org/10.1016/j.psyneuen.2017.06.005

756 Wong, K., Elegante, M., Bartels, B., Elkhayat, S., Tien, D., Roy, S., ... Kalueff, A. V. (2010). Analyzing habituation responses to novelty in zebrafish (Danio rerio). Behavioural Brain Research, 208(2), 450-457. https://doi.org/10.1016/j.bbr.2009.12.023

Writer, B. W., Meyer, E. G., \& Schillerstrom, J. E. (2014). Prazosin for military combat-related PTSD nightmares: A critical review. The Journal of Neuropsychiatry and Clinical Neurosciences, 26(1), 24-33. https://doi.org/10.1176/appi.neuropsych.13010006 
Figure 1

Motor measures of zebrafish in the novel tank test (Experiment 1)

There was a significant effect of time on $(A)$ the total distance traveled per minute and $(B)$ the mean ambulatory speed in the novel tank test ( 6 minutes). All fish generally swam longer distances at faster speeds due to habituation to the novel tank, but there was no effect of 7 days of chronic unpredictable stress and no interaction between stress and time on motor measures ( $\mathrm{N}=25$ in each group).
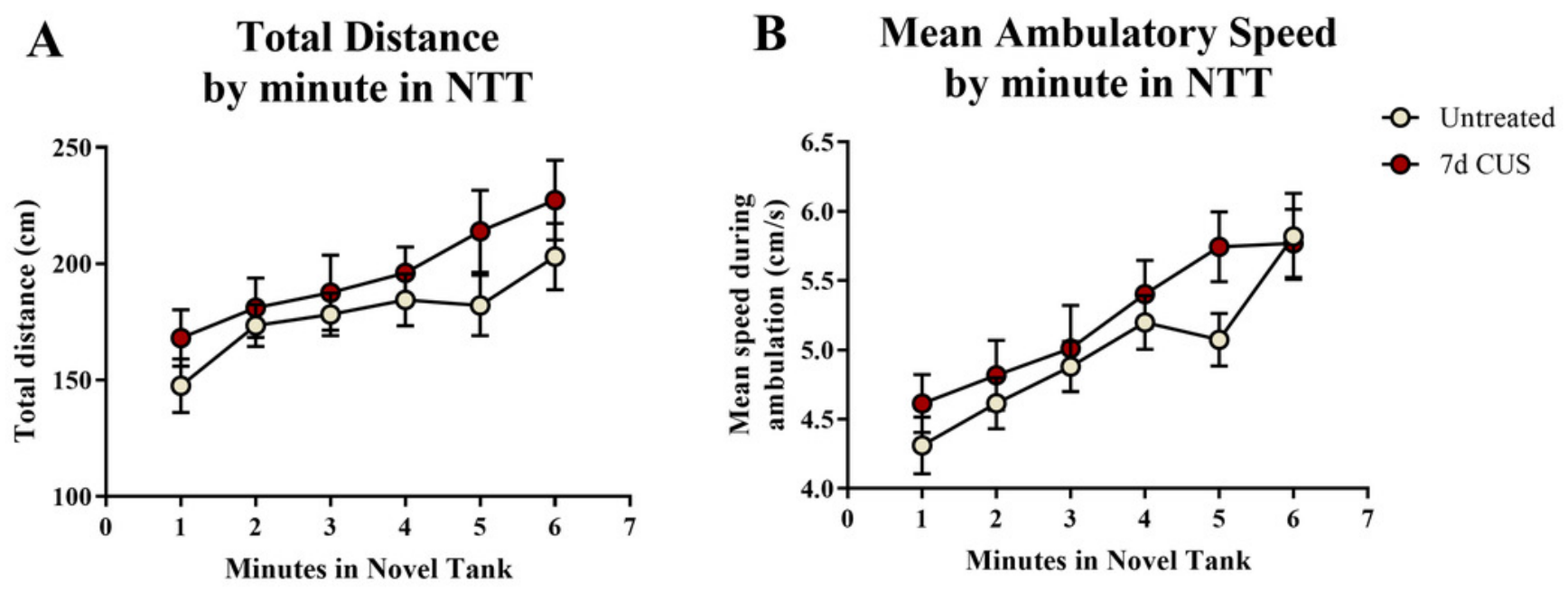
Figure 2

Freezing behavior of zebrafish in the novel tank test (Experiment 1)

There was no effect of treatment or time on the amount of time zebrafish spent immobile in the novel tank test ( 6 minutes, $\mathrm{N}=25$ in each group).

\section{Immobility \\ by minute in NTT}

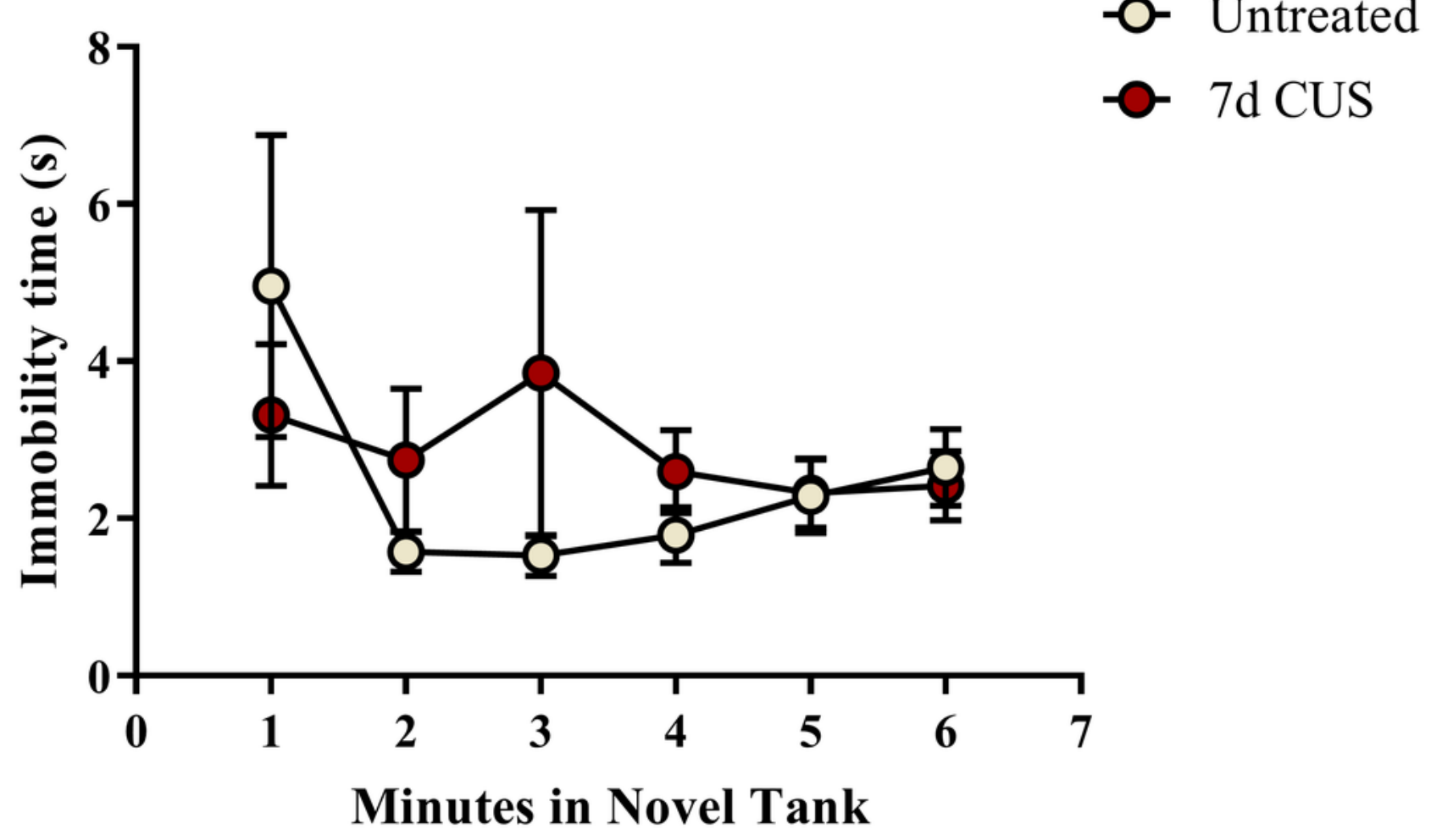




\section{Figure 3}

Exploratory measures of zebrafish in the novel tank test (Experiment 1)

There was a significant effect of time on all top measures in the novel tank test. Additionally, 7 days of chronic unpredictable stress (CUS) (A) marginally decreased the number of times zebrafish entered the top zone, (B) significantly decreased the amount of time zebrafish explored the top zone, and (C) slightly (but non-significantly) decreased the distance traveled in the top of the novel tank test over 6 minutes. Thus, all zebrafish tended to explore the top zone more across the duration of the test; however, chronically stressed fish explored less than non-stressed fish ( $N=25$ in each group).

$\mathbf{A}$

Entries to Top by minute in NTT

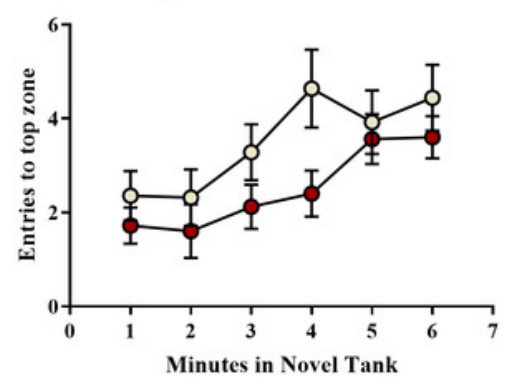

B
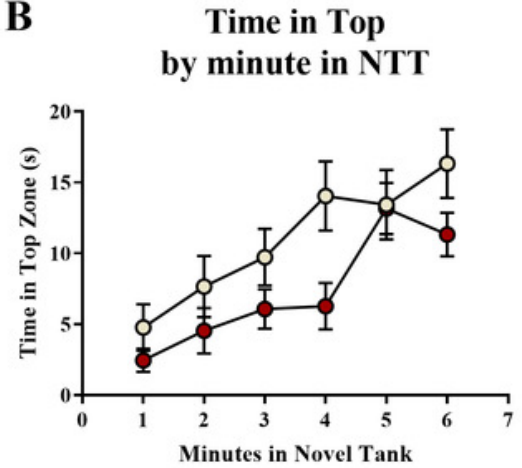

C Distance in Top by minute in NTT

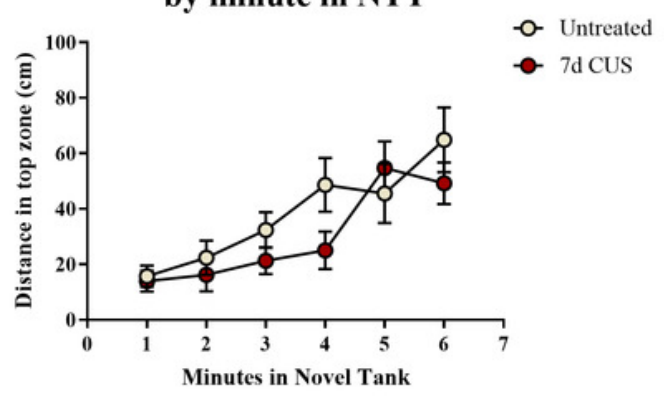


Figure 4

Cortisol and body weight measures of zebrafish (Experiment 1)

Seven days of chronic unpredictable stress (CUS) significantly decreased $(* * p<0.01)$ basal levels of trunk cortisol (A) but did not alter the body weight (B) of zebrafish compared to fish that were left untreated ( $N=25$ in each group).
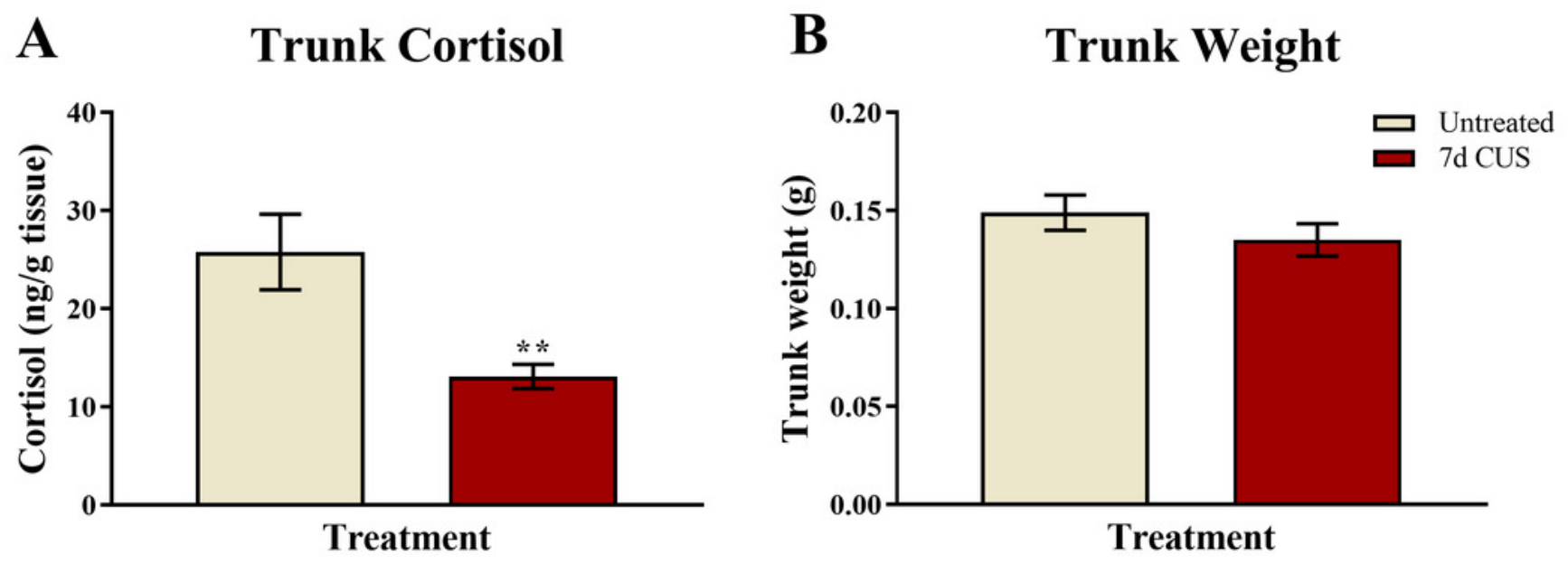
Figure 5

Motor measures of zebrafish in the novel tank test (Experiment 2)

In general, zebrafish gradually increased the total distance traveled per minute (A) and demonstrated increased ambulatory speeds (B) across the duration of the novel tank test (6 minutes), but there was no effect of chronic stress or drug treatment on these measures $(\mathrm{N}=$ 16-18 in each group).
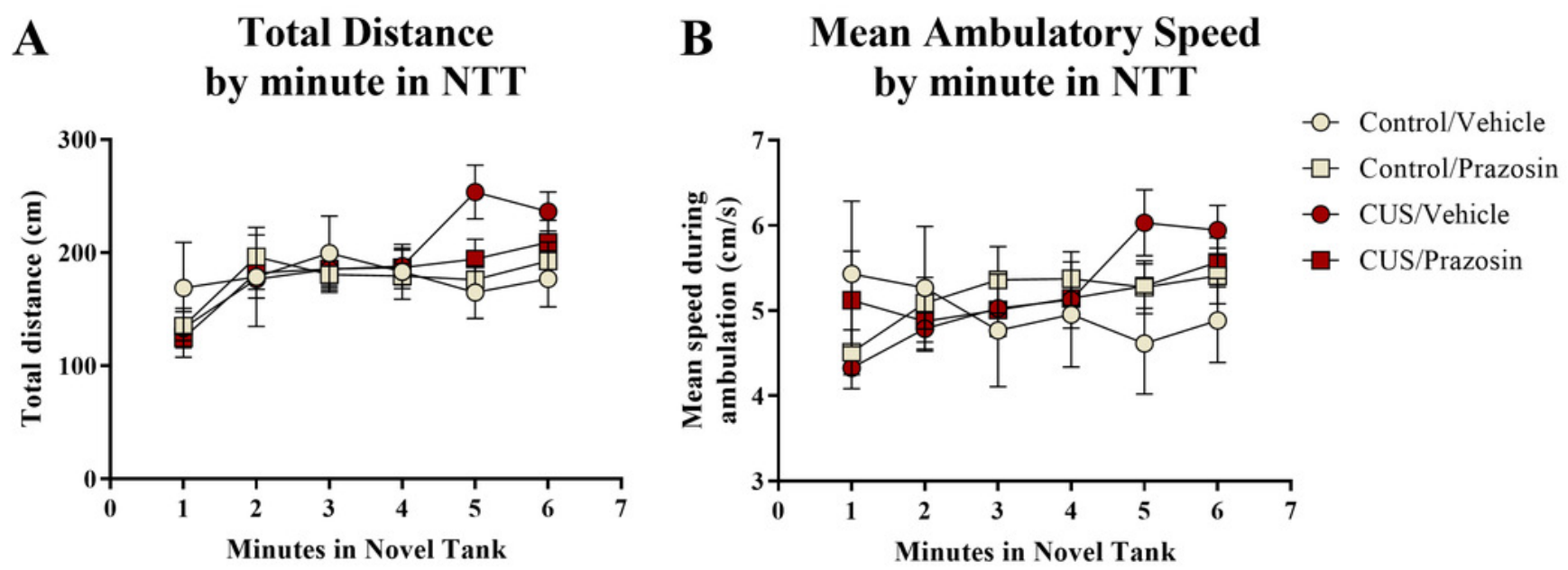
Figure 6

Freezing behavior of zebrafish in the novel tank test (Experiment 2)

In general, zebrafish spent less time immobile across the novel tank test (6 minutes), but

there was no significant effect of chronic stress or drug treatment on immobility ( $N=16-18$ in each group).

\section{Immobility \\ by minute in NTT}

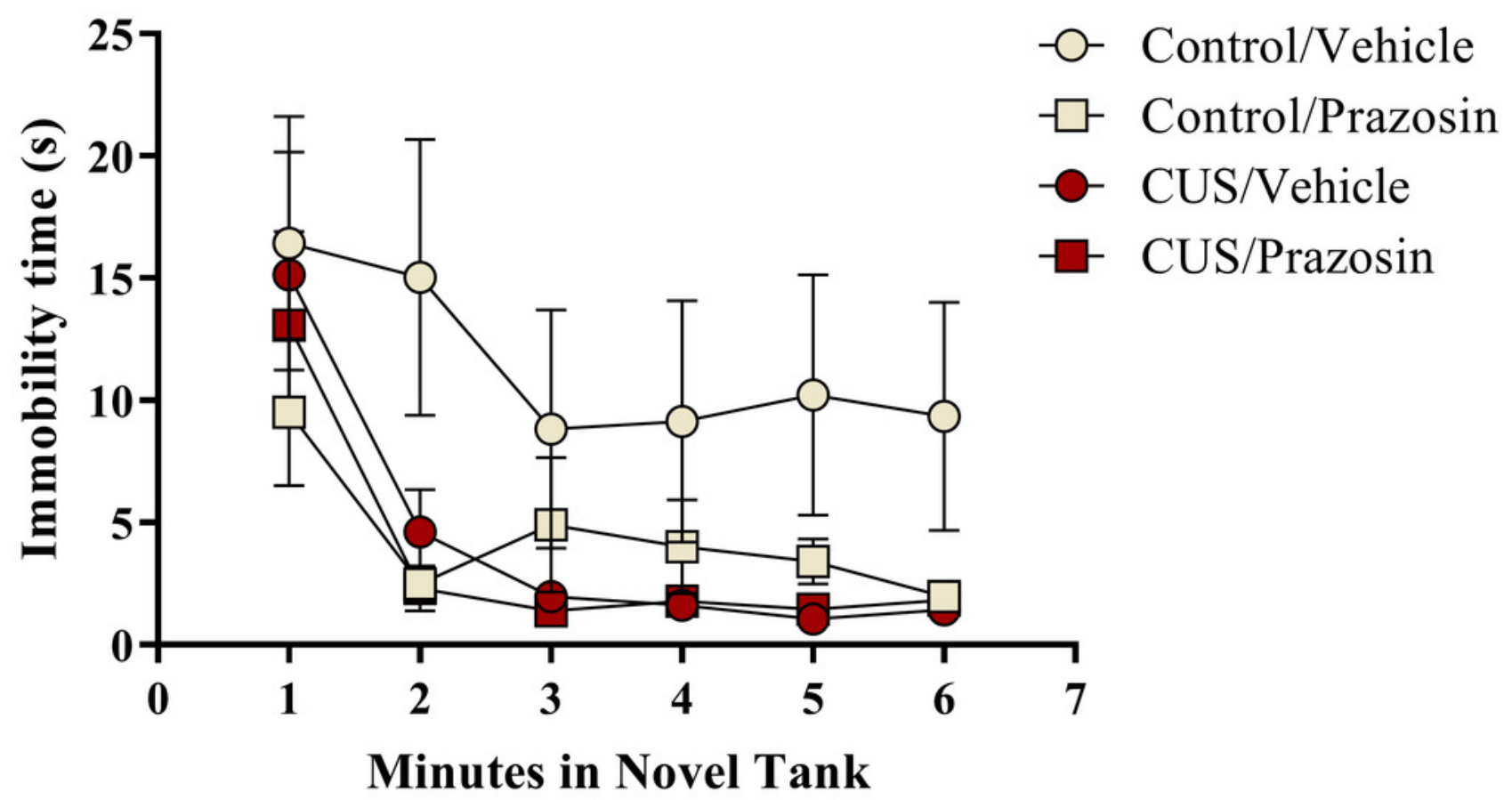




\section{Figure 7}

Exploratory measures of zebrafish in the novel tank test (Experiment 2)

In general, zebrafish explored the top of the novel tank more across the duration of the novel tank test ( 6 minutes) by (A) entering the top zone more frequently, (B) spending more time in the top zone, and (C) traveling a longer distance in the top zone. Zebrafish exposed to chronic unpredictable stress (CUS) for 7 days before drug or vehicle treatments entered the top zone significantly more times and traveled a significantly longer distance in the top compared to non-stressed subjects. If fish were chronically treated with prazosin, they spent significantly more time in the top of the tank compared to vehicle-treated fish $(\mathrm{N}=16-18$ in each group).

$\mathbf{A}$

A

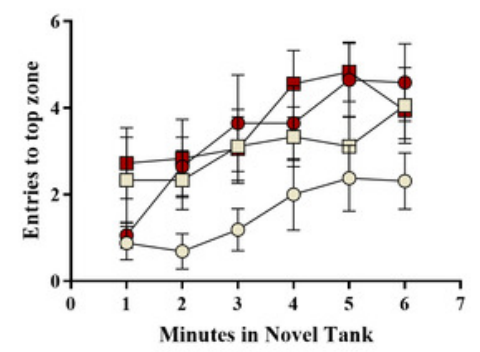

B by minute in NTT

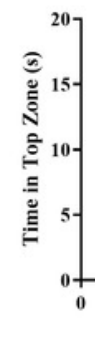

Time in Top

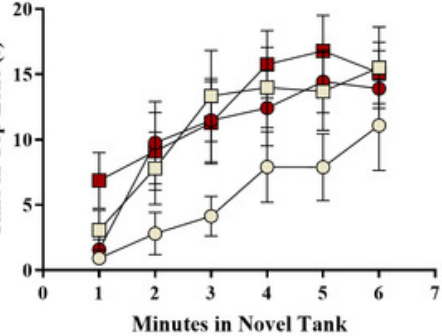

C Distance in Top

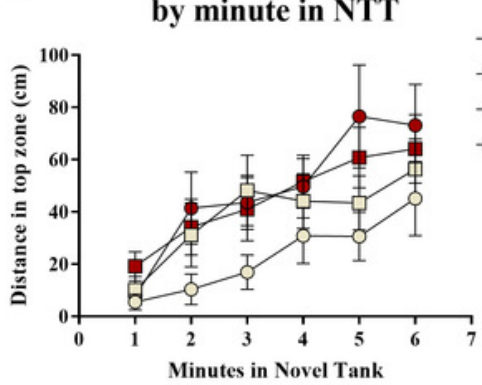

-o- Control/Vehicle

마 Control/Prazosin

- CUS/Vehicle

- CUS/Prazosin 
Figure 8

Cortisol and body weight measures of zebrafish (Experiment 2)

Neither chronic unpredictable stress treatment (CUS) and nor drug treatment significantly altered basal levels of trunk cortisol (A) or body weight (B) of zebrafish ( $N=16-18$ in each group).
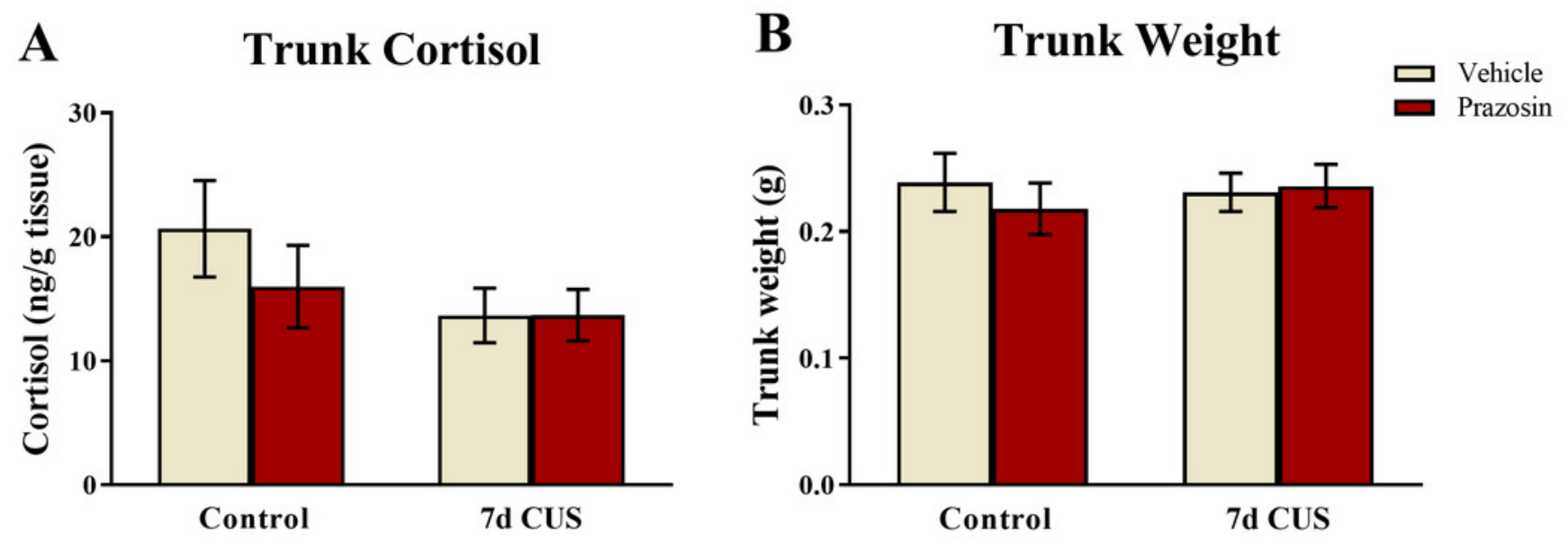


\section{Table $\mathbf{1}$ (on next page)}

Chronic unpredictable stress schedule for Experiment 1 and Experiment 2.

Seven stressors were randomized; fish in the stressed group were exposed to the cycle of stressors twice over 7 days. Subjects were exposed to stressors twice a day at random times during the light period between the times of 9 a.m. and 4 p.m. 
1 Table 1: Chronic unpredictable stress schedule for Experiment 1 and Experiment 2.

2 Seven stressors were randomized; fish in the stressed group were exposed to the cycle of

3 stressors twice over 7 days. Subjects were exposed to stressors twice a day at random times

4 during the light period between the times of 9 a.m. and 4 p.m.

\begin{tabular}{|c|c|c|c|}
\hline $\begin{array}{c}\text { Experiment } \\
\text { Day }\end{array}$ & Stressor & $\begin{array}{c}\text { Time of day } \\
\text { (Experiment 1) }\end{array}$ & $\begin{array}{c}\text { Time of day } \\
\text { (Experiment 2) }\end{array}$ \\
\hline \multirow{2}{*}{1} & Tank changes ( 3 times) & 11 a.m. & 12 p.m. \\
\hline & Cooling $\left(23^{\circ} \mathrm{C}, 30 \mathrm{~min}\right)$ & 1 p.m. & 1 p.m. \\
\hline \multirow{2}{*}{2} & Lowered water (15 min) & 3 p.m. & 2 p.m. \\
\hline & Net chase $(8 \min +15 \min$ rest $+8 \min )$ & 4 p.m. & 3 p.m. \\
\hline \multirow{2}{*}{3} & Crowding ( $250 \mathrm{ml}$ beaker, $60 \mathrm{~min})$ & 10 a.m. & 10 a.m. \\
\hline & Heating $\left(33^{\circ} \mathrm{C}, 30 \mathrm{~min}\right)$ & 2 p.m. & 4 p.m. \\
\hline \multirow{2}{*}{4} & Social isolation ( $250 \mathrm{ml}$ individual beakers, $45 \mathrm{~min})$ & 11 a.m. & 9 a.m. \\
\hline & Tank changes ( 3 times) & 12 p.m. & 11 a.m. \\
\hline \multirow{2}{*}{5} & Cooling $\left(23^{\circ} \mathrm{C}, 30 \mathrm{~min}\right)$ & 3 p.m. & 11 a.m. \\
\hline & Lowered water (15 min) & 4 p.m. & 4 p.m. \\
\hline \multirow{2}{*}{6} & Net chase $(8 \min +15 \min$ rest $+8 \min )$ & 1 p.m. & 11 a.m. \\
\hline & Crowding ( $250 \mathrm{ml}$ beaker, $60 \mathrm{~min})$ & 2 p.m. & 4 p.m. \\
\hline \multirow{2}{*}{7} & Heating $\left(33^{\circ} \mathrm{C}, 30 \mathrm{~min}\right)$ & 10 a.m. & 10 a.m. \\
\hline & Social isolation ( $250 \mathrm{ml}$ individual beakers, $45 \mathrm{~min}$ ) & 12 p.m. & 1 p.m. \\
\hline
\end{tabular}

6 


\section{Table 2 (on next page)}

Overall behavioral measures of zebrafish in the novel tank test (Experiment 1)

Exposure to 7 days of chronic unpredictable stress (CUS) decreased the time spent in the top and marginally decreased the number of times fish entered the top zone of the novel tank in adult zebrafish compared to unstressed (control) fish ( $\mathrm{N}=25$ in each group). 
1 Table 2: Overall behavioral measures of zebrafish in the novel tank test (Experiment 1)

2 Exposure to 7 days of chronic unpredictable stress (CUS) decreased the time spent in the top and

3 marginally decreased the number of times fish entered the top zone of the novel tank in adult

4 zebrafish compared to unstressed (control) fish ( $\mathrm{N}=25$ in each group).

5

\begin{tabular}{lccccccccc}
\hline & \multicolumn{2}{c}{ Control } & \multicolumn{2}{c}{$7 \mathrm{~d}$ CUS } & & & & & \\
\multicolumn{1}{c}{ Variable } & M & SD & M & SD & t & df & p & $\begin{array}{c}\text { Cohen's } \\
\text { d }\end{array}$ \\
& & & & & & & & & \\
& 1082.44 & 225.84 & 1191.60 & 364.03 & 1.274 & 48 & 0.209 & 0.360 \\
\hline Total distance moved (cm) & 5.03 & 0.81 & 5.30 & 0.96 & 1.077 & 48 & 0.287 & 0.305 \\
Mean ambulatory speed (cm/s) & 13.26 & 9.99 & 15.42 & 17.11 & 0.544 & 48 & 0.589 & 0.154 \\
Time immobile (s) & 20.00 & 13.12 & 14.12 & 7.90 & -1.920 & 48 & 0.061 & -0.543 \\
Number of entries to top & 65.96 & 44.75 & 43.82 & 26.49 & -2.129 & 48 & 0.038 & -0.602 \\
Total time in top (s) & 233.58 & 160.16 & 182.32 & 112.61 & -1.309 & 48 & 0.197 & -0.370 \\
Distance in top (cm) & & & & & & & \\
\hline
\end{tabular}

6

7

8

9

10

11 


\section{Table 3 (on next page)}

Overall behavioral measures of zebrafish in the novel tank test (Experiment 2)

In adult zebrafish, exposure to 7 days of chronic unpredictable stress (CUS) increased the distance traveled in the top and the number of top zone entries whereas 7 days of chronic prazosin treatment increased the time spent in the top of zone of the novel tank test $(\mathrm{N}=$ 16-18 in each group). See text for results of significance testing. 
1 Table 3: Overall behavioral measures of zebrafish in the novel tank test (Experiment 2)

2 In adult zebrafish, exposure to 7 days of chronic unpredictable stress (CUS) increased the

3 distance traveled in the top and the number of top zone entries whereas 7 days of chronic

4 prazosin treatment increased the time spent in the top of zone of the novel tank test $(\mathrm{N}=16-18$ in

5 each group). See text for results of significance testing.

6

\begin{tabular}{|c|c|c|c|c|c|c|c|c|}
\hline \multirow[b]{2}{*}{ Variable } & \multicolumn{2}{|c|}{$\begin{array}{c}\text { Control/Vehicle } \\
\mathrm{N}=16\end{array}$} & \multicolumn{2}{|c|}{$\begin{array}{l}\text { Control/Prazosin } \\
\qquad \mathrm{N}=18\end{array}$} & \multicolumn{2}{|c|}{$\begin{array}{l}\text { CUS/Vehicle } \\
\quad \mathrm{N}=17\end{array}$} & \multicolumn{2}{|c|}{$\begin{array}{l}\text { CUS/Prazosin } \\
\quad \mathrm{N}=18\end{array}$} \\
\hline & M & SD & M & SD & M & SD & M & SD \\
\hline $\begin{array}{l}\text { Total distance moved } \\
(\mathrm{cm})\end{array}$ & 1075.90 & 611.65 & 1070.93 & 206.33 & 1162.93 & 349.64 & 1097.22 & 320.95 \\
\hline $\begin{array}{l}\text { Mean ambulatory } \\
\text { speed }(\mathrm{cm} / \mathrm{s})\end{array}$ & 5.83 & 2.60 & 5.21 & 1.07 & 5.31 & 1.05 & 5.13 & 0.96 \\
\hline Time immobile (s) & 67.71 & 108.90 & 24.05 & 28.06 & 24.06 & 27.47 & 19.93 & 18.79 \\
\hline $\begin{array}{l}\text { Number of entries to } \\
\text { top }\end{array}$ & 9.19 & 9.68 & 17.39 & 10.49 & 19.47 & 13.07 & 20.94 & 13.30 \\
\hline Total time in top (s) & 34.78 & 40.07 & 67.42 & 44.41 & 63.59 & 45.87 & 74.91 & 49.61 \\
\hline Distance in top $(\mathrm{cm})$ & 140.69 & 160.39 & 236.92 & 173.72 & 294.23 & 207.33 & 271.54 & 161.64 \\
\hline
\end{tabular}

7 


\section{Table 4 (on next page)}

Results of repeated measures ANOVA (Experiment 2)

Adult zebrafish from all treatment groups generally habituated to the novel tank across the duration of the test ( 6 minutes), but there was no significant interaction between time and drug treatment or stress on any of the behavioral measures of anxiety ( $N=16-18$ in each group). Significance for all dependent variables were determined with a repeated-measures ANOVA with Greenhouse-Geisser correction. 
1 Table 4: Results of repeated measures ANOVA (Experiment 2)

2 Adult zebrafish from all treatment groups generally habituated to the novel tank across the

3 duration of the test (6 minutes), but there was no significant interaction between time and drug

4 treatment or stress on any of the behavioral measures of anxiety $(\mathrm{N}=16-18$ in each group).

5 Significance for all dependent variables were determined with a repeated-measures ANOVA

6 with Greenhouse-Geisser correction.

\begin{tabular}{|c|c|c|c|c|c|c|c|c|c|c|c|c|}
\hline & \multicolumn{2}{|c|}{$\begin{array}{c}\text { Total } \\
\text { distance } \\
(\mathrm{cm})\end{array}$} & \multicolumn{2}{|c|}{$\begin{array}{c}\text { Mean } \\
\text { ambulatory } \\
\text { speed }(\mathrm{cm} / \mathrm{s})\end{array}$} & \multicolumn{2}{|c|}{$\begin{array}{c}\text { Time } \\
\text { immobile (s) }\end{array}$} & \multicolumn{2}{|c|}{$\begin{array}{l}\text { Number of } \\
\text { entries to } \\
\text { top }\end{array}$} & \multicolumn{2}{|c|}{$\begin{array}{l}\text { Total time in } \\
\quad \text { top (s) }\end{array}$} & \multicolumn{2}{|c|}{$\begin{array}{l}\text { Distance in } \\
\text { top }(\mathrm{cm})\end{array}$} \\
\hline & $\mathbf{F}$ & $\mathbf{p}$ & $\mathbf{F}$ & $\mathbf{p}$ & $\mathbf{F}$ & $\mathbf{p}$ & $\mathbf{F}$ & $\mathbf{p}$ & $\mathbf{F}$ & $\mathbf{p}$ & $\mathbf{F}$ & $\mathbf{p}$ \\
\hline \multicolumn{13}{|c|}{ Within-subjects Effects } \\
\hline Time & 9.795 & $<0.001$ & 2.022 & 0.133 & 15.136 & $<0.001$ & 7.112 & $<0.001$ & 15.075 & $<0.001$ & 13.679 & $<0.001$ \\
\hline $\begin{array}{l}\text { Time* } \\
\text { Stress }\end{array}$ & 4.262 & 0.005 & 2.134 & 0.118 & 1.705 & 0.190 & 0.766 & 0.545 & 0.375 & 0.822 & 0.898 & 0.452 \\
\hline $\begin{array}{r}\text { Time* } \\
\text { Drug }\end{array}$ & 0.573 & 0.644 & 0.240 & 0.802 & 0.948 & 0.381 & 0.425 & 0.785 & 0.215 & 0.927 & 0.350 & 0.811 \\
\hline $\begin{array}{r}\text { Time* } \\
\text { Stress* } \\
\text { Drug }\end{array}$ & 1.811 & 0.136 & 3.226 & 0.039 & 0.392 & 0.651 & 0.771 & 0.541 & 0.920 & 0.451 & 0.620 & 0.620 \\
\hline \multicolumn{13}{|c|}{ Between-subjects Effects } \\
\hline Stress & 0.308 & 0.581 & 0.118 & 0.732 & 3.050 & 0.085 & 5.886 & 0.018 & 2.768 & 0.101 & 5.100 & 0.027 \\
\hline Drug & 0.191 & 0.663 & 0.050 & 0.824 & 2.931 & 0.092 & 3.131 & 0.082 & 4.062 & 0.048 & 0.750 & 0.390 \\
\hline $\begin{array}{r}\text { Stress* } \\
\text { Drug }\end{array}$ & 0.100 & 0.753 & 0.123 & 0.727 & 2.018 & 0.160 & 1.431 & 0.236 & 0.956 & 0.332 & 1.876 & 0.176 \\
\hline
\end{tabular}

8 